\title{
THE LEARNING CULTURE SURVEY - PERSISTENCE OF LEARNING CULTURE: RESULTS OF A LONGITUDINAL STUDY ON STUDENTS' PERCEPTIONS OF AND EXPECTATIONS TOWARDS HIGHER EDUCATION
}

\author{
T. Richter \\ University of Applied Sciences Bonn-Rhein-Sieg (GERMANY)
}

\begin{abstract}
The Learning Culture Survey (LCS) is a questionnaire-based research, investigating students' perceptions of and expectations towards Higher Education (HE). The aim of this survey is to improve our understanding about the sources of cultural conflicts in educational scenarios. This understanding, shell help us to predict potential conflict situations and develop supportive measures.
\end{abstract}

After three years of development, the LCS was initialized in 2010 in South Korea and Germany. During the following years, the investigations were extended to further countries. The results, on the one hand, provided insights about the cultural context of HE in general and on the other hand, about specific (national / regional) characteristics of learners in HE. Most issues targeted with the questionnaire were directly linked to value systems. Thus, we expected from the beginning that the collected data would keep valid over longer periods of time. However, we had no evidence regarding the actual persistence of learning culture. For a study, designed to being implemented on a global scope and providing input for further applications, persistence is a basic condition to justify related investigations.

To answer the question on persistence, we repeated the LCS in our university every four years, between 2010 to 2018/19. Besides a small number of slight changes, explainable out of their situational context, the overall results kept consistent over the investigated years. In this paper, after an introduction of the LCS' concept, setting and its general results from the past years, we present the insights from our most recently finalized longitudinal study on learning culture.

Keywords: Higher Education, Learning Culture, Persistence, Longitudinal Study, Learning Culture Survey, Culture in Education.

\section{THE LEARNING CULTURE SURVEY (LCS)}

The motivation for our research originally based on the idea that conflicts during the learning process can lead to learner frustration and thus, reduce the quality of the learning outcomes. Further on, alarming dropout rates were monitored in the context of Higher Education (HE) within the European member states [1] and beyond. We assumed that conflicts, perceived by learners during their learning process, might have played a substantial role for these dropout rates. Thus, being aware of potential conflict sources, could empower us to either generally avoid related clashes through adjusted learning design and preparation of the stakeholders or to design supportive measures for a better handling of conflict situations. Besides rather obvious technological issues, different learning styles, discrepancies between actual and expected knowledge and personal disagreements between students and educators, we found a number of issues in the literature that directly seemed being related to differences between the stakeholders' original culture and the culture in the environment, where they studied or worked. Particularly in international scenarios (e.g., student- and faculty exchange), the found culture-related conflict potential appeared threatening.

We designed the LCS as a questionnaire, developed and standardized over a course of three years and afterwards, in 2010, initially conducted in universities in South Korea and Germany. The results from these studies enabled us to prove that learning culture is not just a phenomenon of academic interest, but an actual source for different types of very tangible conflicts [2]. At least for each of the two, more or less culturally homogenous countries (Germany and South Korea) [3], the students' answers further revealed very similar patterns across investigated universities.

In later investigations, we found limitations of the transferability of our data, in particular, that, even though quite homogenous within the context of $\mathrm{HE}$, learning culture differs between educational contexts: The results of a study, we conducted in the field of German professional training revealed 
significant differences, compared to the results, achieved within the German HE context [4]. In this study, even the results between the investigated German enterprises, already differed enough that the usefulness of a generalization on the level of professional training revealed limited. Regarding professional training, we had to consider the respective organizational cultures as a predominant influence factor.

In a test study, we drove in French and British Cameroon, today one nation but formerly, two separated colonial states (and before that, a large number of regionally distinct societies), we found another limitation for the transferability of the results of our study; even within the context of HE: After conducting an a-priory analysis across the full data-set from Cameroon, $98 \%$ of the responses could correctly be assigned to the one (French Cameroon) or the other (British Cameroon) context as its source. This result must be understood as a strong indicator that "transferring locally achieved results to the whole population of a country that culturally is not homogeneous in itself, is questionable" [5]. Regarding their broad cultural homogeneity, Germany and South Korea must be understood as exceptional cases. However, from our study in the field of professional training in Germany, we already know that we do not deal with a national but a society-specific (or community-specific: HE) phenomenon, which does not necessarily require homogeneity on national, but much more on societal level (see also [6]). For a better understanding, when and to which extent the context of HE is specific enough to assume a dominant community-specific culture, we need a lot more data than currently available (particularly from more countries and more distinct regions within countries).

Between initiating partnerships with supporting universities and accomplishing shareable results, considerable efforts are needed from all sides. Due to the level of automation we already reached, the collection and first analysis of the data requires a relatively low amount of resources (once the online survey is available, which possibly requires its translation, retranslation and implementation). We found that without understanding the investigated context in detail, we can determine whether there are specific characteristics within the investigated context, but we cannot explain them. Anyways, latter is required in order to formulate recommendations or develop measures, supporting learners and educators to deal with culture-specific peculiarities. The solution, we found, was cooperatively analysing the data together with the supporting institutions.

In order to legitimise related efforts for investigations and finally, create a win-win situation for both, our supporting partners and us, the achieved results should prove usefulness from a practical perspective and beyond our own academic interests. A justification can be assumed, if the validity of the collected data remains persistent over a reasonable time. This persistence is a condition that once developed supportive measures do not require re-design with each new students' generation. Up to now, we did not have any evidence that our collected data on learning culture would persist over time.

In the following, we introduce the setting of our longitudinal study and the LCS in general. Afterwards, we present and discuss the results in detail.

\section{GENERAL AND PARTICULAR SETTINGS FOR OUR LONG-TERM STUDY}

For the investigation of the persistence of learning culture, we designed and implemented a long-term study. Every four years between 2010 and 2018/19, we repeatedly conducted the German language version of our online questionnaire at the University of Applied Sciences Bonn-Rhein-Sieg (H-BRS).

We chose these particular intervals, because instead of repetitively receiving responses from the same participants across survey rounds, we wanted to investigate expectations and potential perceptions of students on entry level. The bachelor programs in our university are designed for either six or seven semesters (exception: extra occupational programs). The average of the students finalize their degree within the eighth semester. With this setting, the risk for repeated participations was reduced to students who internally entered master programs, and the low number of exceptionally long studying students.

With the support of our vice presidents for Teaching, Learning and Further Education, we submitted our invitations to the students via the university's general students' e-Mail distribution list. By this means, we ensured that all currently inscribed students were invited for participation and thus, had a chance to make their contribution. The invitation "letters" included a link to the online-questionnaire. Two weeks after the invitation, a second e-Mail was sent as a reminder. The whole survey started a month after the beginning of lectures in the winter semester and was closed six weeks later.

The survey is designed as an anonymous study. We wanted to encourage participants to provide plain answers, even in sensible fields and additionally, circumvent conflicts regarding the protection of 
personalized data. Including the general demographic data, the originally designed questionnaire consists of 106 items, clustered in 12 thematic blocks. For recognition, the English language version of the full questionnaire has permanently been published under the DOI: 10.13140/2.1.2877.5206 [7].

All items within the thematic blocks are mandatory to complete, within the section for demographic data, some inputs are optional to provide. Most of the thematic items are designed as statements on opinions, which the respondents are to evaluate on a Likert scale between disagree (1) and fully agree (4). We rather wanted to enforce the respondents' positioning instead of offering a neutral answer opportunity. Alternatively, we provide an additional fifth opportunity for the cases that a certain statement simply cannot be applied to the particular context of the respondent. This fifth field appears visually isolated from the regular scale. This design was a compromise to avoid dropouts due to conflicts between having to and not being able to provide an answer on a specific item. In the past studies, the respondents rarely made use of this option.

In order to detect randomly completed questionnaires, we implemented three test items, repeating previously made statements.

The twelve thematic blocks are:

1 Role of the Professor / Lecturer

2 Tasks and Responsibilities of the Professor / Lecturer

3 Feedback

4 Motivation

5 Gender Issues

6 Group Work Efficiency

7 Where Group Work supports my own Learning Process

8 Group Building Process

9 Open Statements

10 Time Management

11 Role of the Tutor

12 Tasks of the Tutor

From the design, each deck of the online-questionnaire covers one of the above thematic blocks. The demographic data are to be collected in the end. For completion, the design of the online-questionnaire enforces the processing of all mandatory fields. As basic condition for the evaluation, we consequently defined a $100 \%$ completion rate of mandatory items (everything else are considered invalid drop-outs).

Before the actual data-evaluation can start, the samples need to be cleaned and "normalized": In a first step, any incomplete responses are to be excluded (deleted) from the sample. Further, all responses are to be excluded, in which the answers of the three test items do not match the expected values (we yet had no such case). In the next step, the textual responses in the demographic section need to (manually) be harmonized, so that spellings, acronyms or alternative wordings do not lead to errors in the later data analysis (e.g. "Hochschule Bonn-Rhein-Sieg", Hochschule Bonn Rhein Sieg, HS BonnRhein-Sieg”, University of Applied Sciences Bonn-Rhein-Sieg,"H-BRS”, "HBRS” become "HBRS”).

We expected having to deal with very different sample sizes, which made the direct comparison of different data-sets complicated. For ordinal scaled data, Bauer [8] recommends to circumvent the negative effect of different sample sizes through binarizing. Following this approach, we subsume our data in disagreements (negative answers, $1 \& 2$ ) and agreements (positive answers, $3 \& 4$ ) and calculate percentage values for positive/negative answers related to the full sample. The so subsumed detailed information is still used to calculate median and 40/60 quantiles: In some cases of development or differences between contexts, the measures of location proved helpful for the interpretation.

For the comparison of data-sets, we focus on the percentage of positive answers. The results from each thematic block are collectively displayed within a distinct net diagram. As visible outcome, the data lead to a sample-specific pattern. It is characterized through the number of items of the represented thematic block, defined through the number of axes which span the space for the net and through each item's percentage value, which defines one point on the assigned axis. The visible patterns eventually result 
from connecting the defined points with those on their neighbouring axes. When having to contrast samples, these patterns allow to quickly recognize differences on a visual basis.

During the operationalization phase of the questionnaire, we adopted the culture-definition from Oetting [9], who understands culture as "the customs, beliefs, social structure, and activities of any group of people who share a common identification and who would label themselves as members of that group".

With this concept of culture as something, the majority of the members within a society has in common, the central question emerged, on how to distinguish culturally motivated from rather individually different responses. We expected that just culturally influenced issues would lead to data which are persistent over a longer period of time (and thus, interesting for later applications), because individual opinions could easily be re-evaluated and changed, e.g., due to new information. Statistically, any 50/50 [\%] distribution (negative/positive) signifies individual differences, while any other distribution, a majority and thus, a culturally motivated decision. However, such a sharp distinction between individually and culturally motivated responses cannot live up with the instable reality of individual opinions. In order to obtain more certainty, we decided to implement a broader "fuzzy zone", in which every result within the corridor above 40 and below 60 percent positive/negative answers, is to be interpreted as individual differences (without further potential for culture-related interpretation). Any other result indicates a cultural background. As the distance between responses, considered large enough for a reasonable interpretation of a substantial difference between contexts, we decided defined ten percent (everything below is "just" a bit more or less).

\section{RESULTS}

In the following, we present the results of our longitudinal study according to each thematic block of the questionnaire, using net-diagrams.

\subsection{The Role and Tasks of the Lecturer}

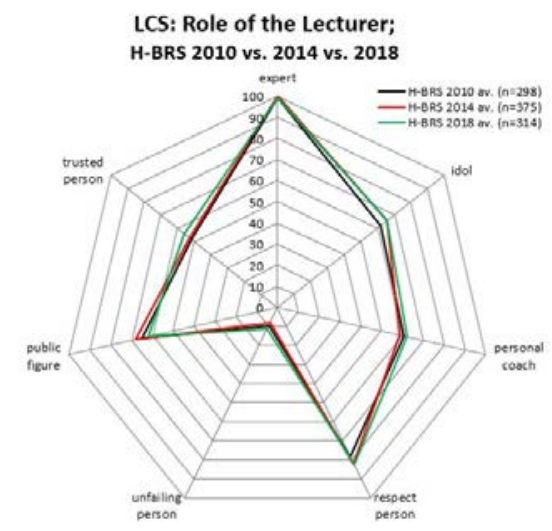

Figure 1. The Role of the Lecturer.

Over the course of eight years, neither the role (Fig. 1), nor the tasks (Fig. 2), assigned to a lecturer in $\mathrm{HE}$, changed significantly in the view of the participating students.

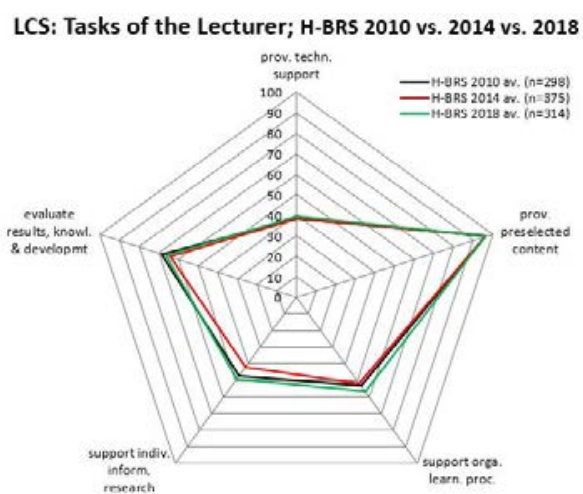

Figure 2. The Tasks of the Lecturer. 


\subsection{Feedback}

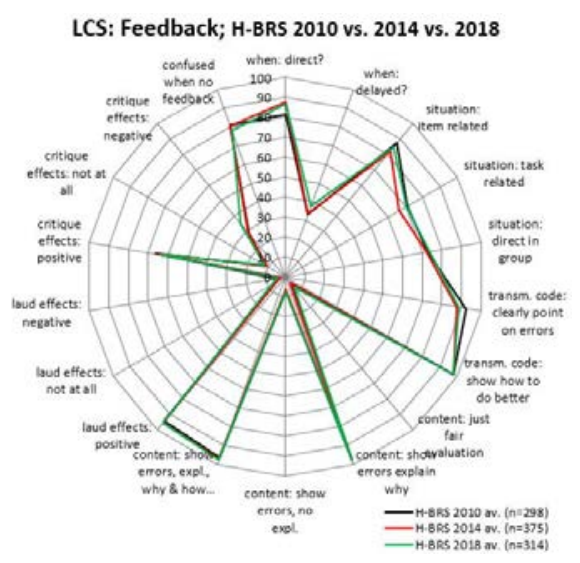

Figure 3. Feedback.

The students' perceptions of and expectations towards feedback kept almost unchanged over the period of eight years (Fig. 3).

\subsection{Motivation}

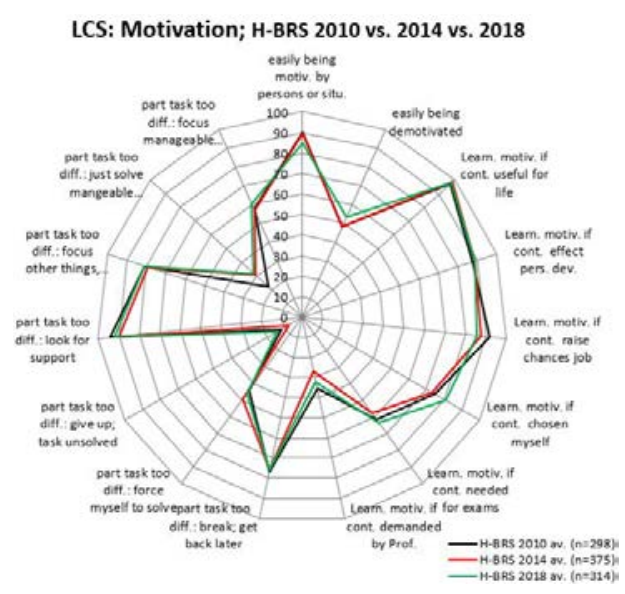

Figure 4. Motivation.

Regarding the students' perceptions of motivation and their strategies to deal with very challenging tasks (Fig. 4), no remarkable differences were monitored between the results of the studies from 2010, 2014 and $2018 / 9$.

\subsection{Gender issues}

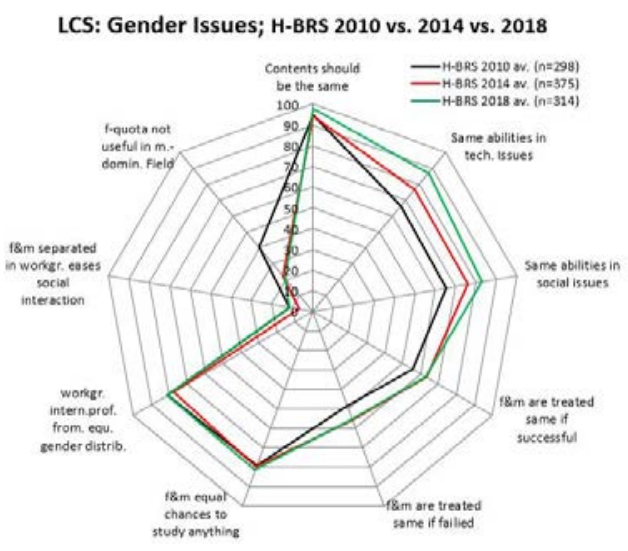

Figure 5. Gender Issues. 
In the section "Gender Issues" (Fig. 5), we found a constantly increasing number of positive evaluations regarding the (general) assessment of gender-specific qualification in certain fields (technical / social issues). Further on, in the studies from 2014 and 2018/9, the students clearly showed a different perception regarding the concept of and necessity for a female quota in male-dominated fields. This change happened in the same period, in which aspects of political correctness, gender consciousness and gender equality increasingly became a focus of public debates in Germany. During the analysis from 2014, we found that this particular change directly reflected a shift in the understanding (and evaluation) of the different facets of gender equality within the German society [5].

\subsection{Group Work efficiency}

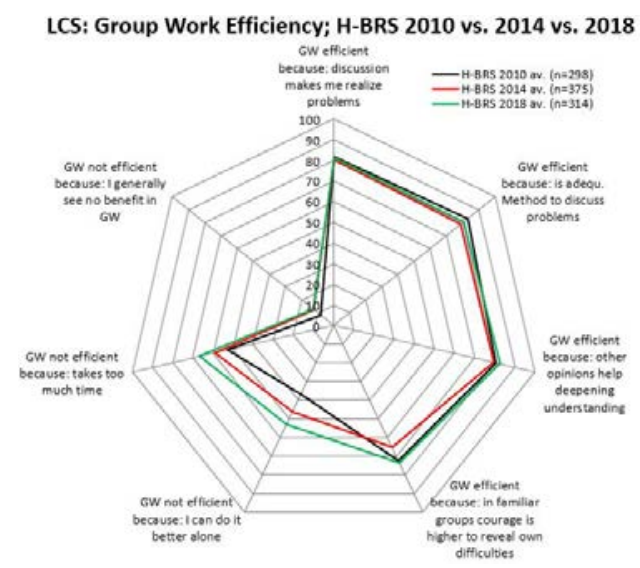

Figure 6. Group work efficiency.

In 2010, more than 50 percent of the students stated that they rather do not like to work in groups, because they considered individual work much more efficient. From 2014, the acceptance of group work (GW) constantly increased, up to $62 \%$ of the students, in 2018/9 (see Fig. 6). It seems, the students started to recognize a benefit in GW. The German society generally is considered individualistic, and so, the result from 2010 was quite expectable, particularly since it anyways was located within the fuzzy area of our scale (individually different responses). The monitored change in 2014 could possibly reflect the increasing demands, students had to face, alongside with the application of much more project work within the diverse university courses. Since some years, lectures increasingly shift from the traditional behaviorist to cognitivist and even constructivist education, which must be understood as a change in university culture. However, to determine if we actually face a trend, requires further investigations.

\subsection{Perceived benefits of voluntary GW and group building process}

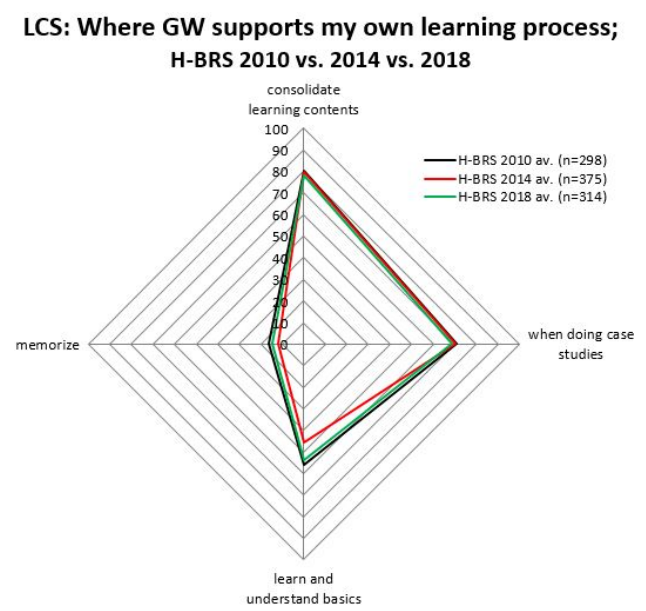

Figure 7. Where GW supports my own learning process. 


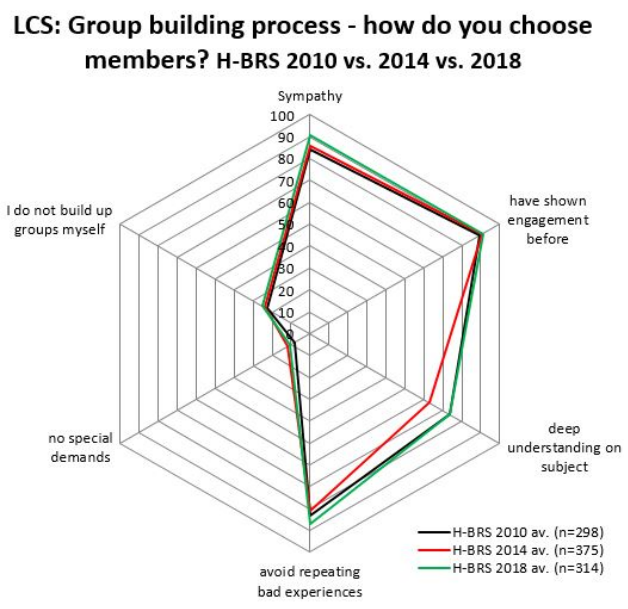

Figure 8. Group Building Process.

Regarding the perceived benefits of voluntary GW (Fig. 7), no significant changes were found across the three samples. However, German students appear to increasingly learn and discuss basics within teams. The decision to do so, still is an individual one (fuzzy corridor), but the noticeable development also confirms our assumption from Figure 6 . As a very different example, the students from South Korea, which is considered a collectivist society, provided $100 \%$ positive responses across over 30 universities and for all four items. Regarding the group building process (Fig. 8), no remarkable trends were found across the samples.

\subsection{Diverse Issues of (instructed) GW and Time Management}

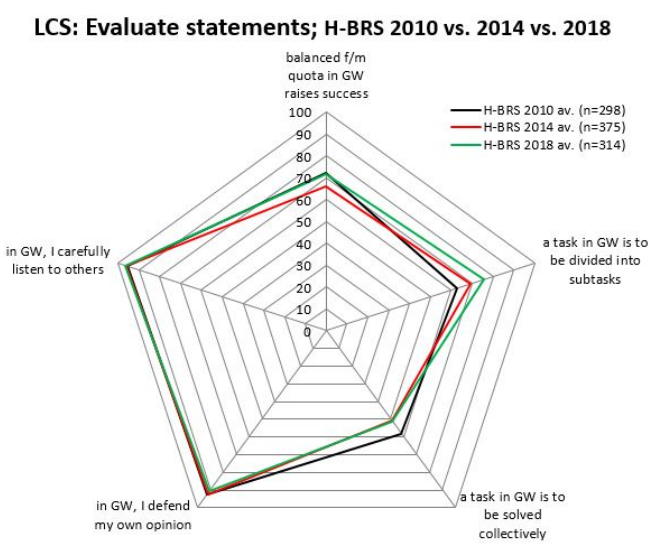

Figure 9. Diverse Issues of GW.

In Fig. 9, no significant changes were found, but the answers on two of the items still appear remarkable: Over the course of eight years the students increasingly demanded that tasks within instructed GW should be dividable into distinct subtasks. One could assume the German students have resigned, since they have to solve tasks in teams but fear that their individual pace could be disturbed, because of dependencies from other work results. Somehow in contrast, while in 2010 and 2014 the students were discordant, if evaluations on results of GW should be given on the basis of individual or collective results, there appears to be an increasing willingness to take responsibility for the whole group (we cannot speak of a trend yet, and still, the responses lay in the fuzzy area, but the issue should further be monitored). 


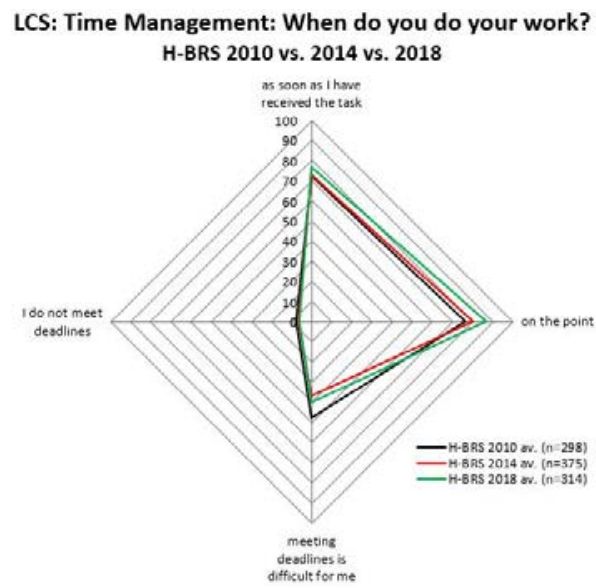

Figure 10. Time Management.

In the thematic block "time management" (Fig. 10), no significant changes were found. However, students increasingly report that they finalize their work on the point, which somehow confirms the priory taken assumption that studying (and thus, time management) gets tougher for them.

\subsection{The Role and Tasks of the Tutor}

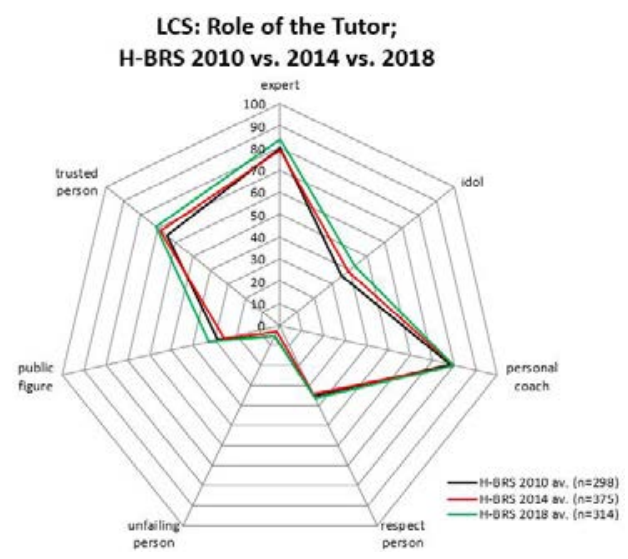

Figure 11. The Role of the Tutor.

In German universities, tutorials, traditionally are held by students of higher semesters (or doctoral students). Thus, the perceived role and tasks of lecturers significantly differs from the results in this thematic block (in Germany). Across the investigations from 2010, 2014 and 2018/9, no significant changes were found in either the perceptions of the role (Fig. 11) or the expectations towards the tasks (Fig. 12) of tutors.

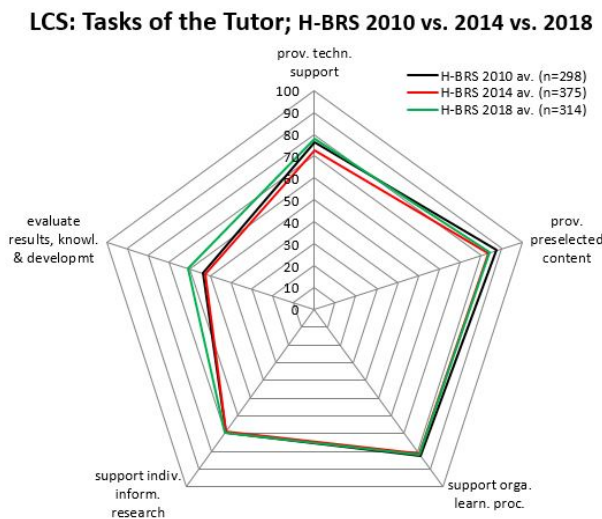

Figure 12. The Tasks of the Tutor. 
As an example for a very different result, in South Korea, tutorials usually are provided by the professors (lecturers), so that between the expectations towards lectors and tutors no significant difference was found. This, particularly is a good example that a reasonable interpretation of found results, in many cases, is sheer impossible without a deeper understanding of the internal settings and mechanisms within the investigated context.

\section{CONCLUSIONS}

Across the three investigations with German students from 2010, 2014 and 2018/9, most issues kept unchanged. We can take the following conclusions:

Even though learning culture, as we designed its' concept, is not static, it still is quite persistent over time. This confirms the general results of the diverse disciplines driving value-based culture research. Value shifts in the investigated society or in the particular context of the investigated educational scenario, result in changes regarding learning culture. The changes we eventually found between the samples of the three investigations, were minor in dimension, but could become trends. In all cases, we were able to find sound explanations linked to either cultural changes in the German society in general, or within university culture and changed study-conditions, in particular.

The result of the longitudinal study confirms our concept of learning culture and assigns our data the persistence over time, required to justify the efforts, for a widening of the scope of data collection to international level. It further appears reasonable to focus on more practice-oriented research.

The Leaning Culture Survey was never designed as a singular project with a defined budget and timeframe, but instead, focuses on ongoing, collaborative work across universities and countries. In the past years and in order to report about basic findings on the characteristics of learning culture, our work results had exclusively been shared through publications and invited presentations and lectures.

With the widening of our scope and related results, we plan to develop a publicly available, web-based application. This data-base driven application shall give all interested users a chance, to study the available data according to individually defined selection criteria (country/region/thematic block). They further will be able to compare results across two selected contexts and, as far as possible in the future, also receive hints on conflict potential and so far successful interventions. As typical users for our application, we see learners and educators (in preparation for a planned study or work abroad), offices for international affairs (e.g., our data can be considered in their counselling work) and educators / researchers who try to better understand (and predict) conflicts in culturally diverse learning scenarios. For learners, studying abroad, we expect that our research results can make a significant difference between experienced cultural diversity and perceived culture clashes.

\section{CALL FOR PARTICIPATION, REQUIRED EFFORDS AND POSSIBLE LEVELS OF CONTRIBUTION}

With this paper, we would like to invite and encourage universities all over the world to participate in this research. What needs to be done from your perspective?

For the data collection, the main work for partners can be reduced to distributing the invitation-e-Mail with the included survey-link to the students. For the quality of the data, it is relevant to invite all students, currently inscribed in the university. Just involving a faculty or even a course might be good enough for a test study but does not meet the requirements for representative data. The alternative would be defining and (randomly) selecting a representative group of students, which we experienced is sheer impossible on various levels. In the past, we achieved best successes, when the e-Mail with the link to the online questionnaire was internally sent by a person, prominent across faculties, such as, the Vice Presidents for Teaching, Learning and Further Education at the H-BRS. Generally, the questionnaire shall always be provided in the official national language. If the required language version of the questionnaire already is available, we can manage to provide the link to the questionnaire within two weeks, counted from the decision to conduct the survey. As survey-platform, we use Unipark, a software, produced by Questback. It has been implemented for the special needs of academic research and is provided and hosted in Berlin, Germany.

Two weeks after the first invitation, a reminder e-Mail is to be sent to the students (also internally within the investigated university). Given that at least $3 \%$ of the students responded, the questionnaire can be closed after the first reminder; else, a second reminder should be sent. We experienced, sending the 
invitations to the students at the beginning of a semester, was the best strategy, so far. After the second reminder was sent, the "job" of the partner university is done (in terms of data collection). Six weeks from start, the questionnaire is being closed (a defined and communicated time out). We download the data from the survey-platform provider, clean and normalize the dataset and conduct the analysis. The raw data (an Excel File) are to be sent to the partner institution, alongside with the comparative analysis (with the German sample).

For the interpretation, we initially compare the recently collected data with our (consolidated) data from Germany, in order to set a starting point, from which we determine differences, which require contextspecific explanations. In the past, we followed two different strategies for the next step:

1 We presented the data (in person) within the partner universities, either in form of a (frontal) lecture, with students and lecturers as participants and open discussions in the end, or as a workshop, with mainly lecturers as participants. During the workshops, we jointly analysed the results: For each item, we first explained the findings according to our home-context and afterwards, asked the local participants for possible reasons for recognized differences. In the end, we further discussed possible conflicts and strategies to prevent/overcome them. Since there was no funding available, in both upper scenarios, travel and accommodation expenses were financed by the inviting institutions (in one case, partners visited us).

2 As alternative setting, we transferred the results of our comparative analysis (including questions) into presentation slides, sent them alongside with the raw data to the partners and organized online discussions. While it was possible to save travel expenses taking this approach, the results were less comprehensive.

As for the availability of translations, if the questionnaire is already available in the respective language version, no further steps are required. In the cases, the respective language versions are not yet available or the translations not yet verified, we may need further support from our partners. Currently, each available language version has either been translated by professional translators (Portuguese, French, trad. Chinese and Mandarin, Japanese) or been translated from English and retranslated to English, each by native speakers (Korean, English). The available language versions following our quality standard, are ready to check (e.g., for political correctness), implement and use. We additionally have language versions in Bulgarian, Russian and Turkish. These versions have been translated by students, but are not yet verified (retranslated and corrected).

We would like to encourage universities from all over the world to collaborate with us. Data are needed from every country in the world, and having the chance to investigate more than a single university within a country / region would enhance the quality of data for and knowledge about the respective country.

While our future research will focus on $\mathrm{HE}$, we also still welcome invitations to drive studies within other learning contexts, such as large enterprises and NGOs.

\section{REFERENCES}

[1] H. Vossensteyn, A. Kottmann, B. Jongbloed, F. Kaiser, L. Cremoni, B. Stensaker, E. Hovdhaugen, \& S. Wollscheid, „Dropout and Completion in Higher Education in Europe“, Education and Culture, European Commission, 2015. Retrieved from https://supporthere.org/sites/default/files/dropoutcompletion-he_en.pdf

[2] T. Richter, "Culture-Specific Perceptions of Motivation and Implications for Technology Enhanced Learning", European Journal of Open, Distance and E-Learning (EURODL), "Special Issue: Best of Eden 2013/14", pp. 99-111, 2015.

[3] P. Ziltener, "Societal Heterogeneity in Africa and Asia: A Comparative Analysis of Its Impact on Development", Zeitschrift für Soziologie, number 35, issue 4, pp. 286-304, 2006.

[4] T. Richter \& H. Adelsberger, "On the myth of a general national culture: Making specific cultural characteristics of learners in different educational contexts in Germany visible", In: M. Strano, F. Sudweeks, H. Hrachovec \& C. Ess, (Eds.), Part 1 of the CATaC'12 Proceedings, School of Information Technology, Murdoch University, Murdoch, Australia, pp.105-120, 2012.

[5] T. Richter \& A. Zelenkauskaite, "Culture, Gender and Technology Enhanced Learning: Female and Male Students' Perceptions across three Countries". In: M.B. Nunes, \& M. McPherson (Eds.), Proceedings of the $8^{\text {th }}$ IADIS International Conference e-Learning 2014 (MCCIS), IADIS Press, Lisbon, Portugal, pp. 3-12, 2014. 
[6] P. Leonardi, "Cultural variability and web interface design: Communicating US Hispanic cultural values on the Internet", in F. Sudweeks \& C. Ess (eds.), CATaC'02 Proceedings, Murdoch University: Murdoch, Australia, pp. 297-316, 2002.

[7] T. Richter, "The Learning Culture Survey: An international research project on cultural learning attitudes; English language questionnaire version for recognition". Due-Publico, Essen, 2014.

[8] N. Baur, „Das Ordinalskalenproblem“, in N. Baur \& S. Fromm (Eds.), Datenanalyse mit SPSS für Fortgeschrittene, $2^{\text {nd }}$ ed., Wiesbaden: VS Verlag, pp. 279-289, 2008.

[9] E.R. Oetting, "Orthogonal Cultural Identification: Theoretical Links Between Cultural Identification and Substance Use", in: M.R. de la Rosa \& J.-L.R. Andrados (Eds.), Drug Abuse Among Minority Youth: Methodological Issues and Recent Research Advances, DHHS/PHS, Rockville, MD, pp. 3256, 1993. 